\title{
Fault Detection and Diagnosis of Winding Short in BLDC Motors Based on Fuzzy Similarity
}

\author{
Hyeon Bae*, Sungshin Kim** and George Vachtsevanos* \\ *School of Electrical and Computer Engineering, Georgia Institute of Technology \\ **School of Electrical and Computer Engineering, Pusan National University
}

\begin{abstract}
The turn-to-turn short is one major fault of the motor faults of BLDC motors and can appear frequently. When the fault happens, the motor can be operated without breakdown, but it is necessary to maintain the motor for continuous working. In past research, several methods have been applied to detect winding faults. The representative approaches have been focusing on current signals, which can give important information to extract features and to detect faults. In this study, current sensors were installed to measure signals for fault detection of BLDC motors. In this study, the Park's vector method was used to extract the features and to isolate the faults from the current measured by sensors. Because this method can consider the three-phase current values, it is useful to detect features from one-phase and three-phase faults. After extracting two-dimensional features, the final feature was generated by using the two-dimensional values using the distance equation. The values were used in fuzzy similarity to isolate the faults. Fuzzy similarity is an available tool to diagnose the fault without model generation and the fault was converted to the percentage value that can be considered as possibility of the fault.
\end{abstract}

Key Words : BLDC motor, fault detection and diagnosis, Park’s vector, fuzzy similarity

\section{Introduction}

The most popular way of converting electrical energy to mechanical energy is an induction motor. This motor plays an important role in modern industrial plants. The risk of motor failure can be remarkably reduced if normal service conditions can be arranged in advance. In other words, one may avoid very costly expensive downtime by replacing or repairing motors if warning signs of impending failure can be headed. In recent years, fault diagnosis has become a challenging topic for many electric machine researchers. The major faults of electrical machines can be broadly classified as follows [1]:

- Broken rotor bar or cracked rotor end-rings

- Static and dynamic air-gap irregularities

- Dynamic eccentricity

- Winding short

- Bearing and gearbox failure

The diagnostic methods to identify the faults listed above may involve several different types of fields of science and technology [1-3]. Many types of signals have been studied for the fault detection of induction motors. However, each technique has advantages and disadvantages with respect to the various types of faults. Several methods used to detect faults in induction motors are as follows:

- Electromagnetic field monitoring

- Temperature measurements

- Radio frequency (RF) emissions monitoring

- Noise and vibration monitoring

Manuscript received Jan. 14. 2009; revised May. 25. 2009.

This work was supported by the Korea Research Foundation Grant funded by the Korean Government (KRF-2007-357D00092).
- Motor current signature analysis (MCSA)

- $\mathrm{AI}$ and $\mathrm{NN}$ based techniques

In this study, winding short of the BLDC motor was detected and isolated. The applied faults are typical and often occurred types in industrial fields. The signal for winding short was analyzed based on MCSA. To extract features from the current signals, the vector-based approach was used to detect the fault pattern from the raw data. The Park's vector method was employed for the vector-based analysis [4-7]. This approach uses three-phase signals for feature extraction. In this approach, the three-phase signals are transformed to two-dimensional values. For the features in this study, the distance of the twodimensional values was calculated; therefore, the final results are expressed by one-dimensional values.

After extracting the features, fuzzy similarity was applied to isolate the fault. Fuzzy similarity can be easily used without using a model [8-11] and it is useful to express the possibility of the fault as similarity measure. In actual, fuzzy similarity measure was available to isolate the fault based on the fault possibility.

\section{Fault Detection and Isolation}

\subsection{Park's Vector}

Park's vector transforms three-phase current or voltage signals to two dimensions ( $d-q$ domain). Because a time-based signal is changed to an angular frequency signal, it can be easier to be analyzed [4-7]. This method has been widely applied in fault detection of the motor. Usually it is used in brushless DC motors, because current signals of the BLDC motors are periodic and sign curves. The result of Park's vector 
is illustrated as a circle when the signal is normal case signal. The current signal of the BLDC motor is not an exact sign curve, but this approach is also useful in fault detection of the BLDC motor. The online diagnosis is based on identifying the appearance of an elliptical pattern, corresponding to the motor current Park's Vector representation, whose ellipticity increases with the severity of the fault and whose major axis orientation is associated with the faulty phase. This result means that the fault phase signal can affect the shape of the $d-q$ domain value. Therefore, the fault signal can be detected by the shape that is transformed by Park's vector. As a function of mains phasevariables $\left(i_{A}, i_{B}, i_{C}\right)$ the current Park's Vector components $\left(i_{D}\right.$, $i_{Q}$ ) are

$$
\begin{gathered}
i_{D}=\frac{\sqrt{2}}{\sqrt{3}} i_{A}-\frac{1}{\sqrt{6}} i_{B}-\frac{1}{\sqrt{6}} i_{C} \\
i_{Q}=\frac{1}{\sqrt{2}} i_{B}-\frac{1}{\sqrt{2}} i_{C}
\end{gathered}
$$

\subsection{Feature Extraction}

As mentioned above, the Park's vector approach makes a feature signal using three-phase current signals. This method can observe the variation of each phase of currents, so it is useful to detect faults related to the amplitude and phase variation. In this method, 3-phase currents $\left(i_{a}, i_{b}, i_{c}\right)$ are used for fault dictation that is different with other two approaches that use time-domain (envelop method) and frequency-domain (FFT). Two dimensional values $\left(i_{D}, i_{Q}\right)$ were calculated to one dimensional signal by distance calculation. As shown in Eq. (3), finally, the vector-based values (two-dimension) were expressed as distance values (one-dimension) for fault detection.

$$
i_{\text {Feautre }}=\sqrt{\left(i_{D}^{2}+i_{Q}^{2}\right)^{2}}
$$

\subsection{Fault Isolation}

To diagnose faults from the features extracted by using Park's vector, fuzzy similarity was applied [8-11]. The features can be represented by a function of a fuzzy membership function. As shown in Eq. (4), a fuzzy membership function can be expressed as unit amplitude of a Gaussian function. The fuzzy membership function can be used for fault isolation. In the fuzzy membership function, $\mathrm{c}$ and $\mathrm{b}$ mean the standard deviation and average value, therefore both parameters can be calculated by using the experimental data.

$$
\begin{aligned}
& f(x)=a \exp ^{-\frac{(x-b)^{2}}{2 c^{2}}}: \text { Gaussian Function } \\
& \Rightarrow \mu(x)=\exp ^{-\frac{(x-b)^{2}}{2 c^{2}}}: \text { Fuzzy Membership Function }
\end{aligned}
$$

As shown in Fig. 1, fuzzy similarity can be measured by using fuzzy membership functions $A$ and $B$ as shown in Eq. (5). This equation calculates fuzzy similarity between two membership functions. The fault condition can be isolated based on the baseline function with the similarity.

$$
M_{A, B}=\frac{|A \cap B|}{|A \cup B|}=\frac{\sum_{i=1}^{n}\left(a_{i} \wedge b_{i}\right)}{\sum_{i=1}^{n}\left(a_{i} \vee b_{i}\right)}
$$

However, when the degree of the similarity is lower, the possibility of the fault is higher. Therefore, in this study, the concept of dissimilarity was used for fault isolation. The dissimilarity degree can be expressed as 0 to 1 . If this degree is converted to the percentage value, the value can be fault severity as 0 to $100 \%$. By using the percentage value, the fault can be isolated.

$$
D M_{A, B}=1-\frac{|A \cap B|}{|A \cup B|}=1-\frac{\sum_{i=1}^{n}\left(a_{i} \wedge b_{i}\right)}{\sum_{i=1}^{n}\left(a_{i} \vee b_{i}\right)}
$$
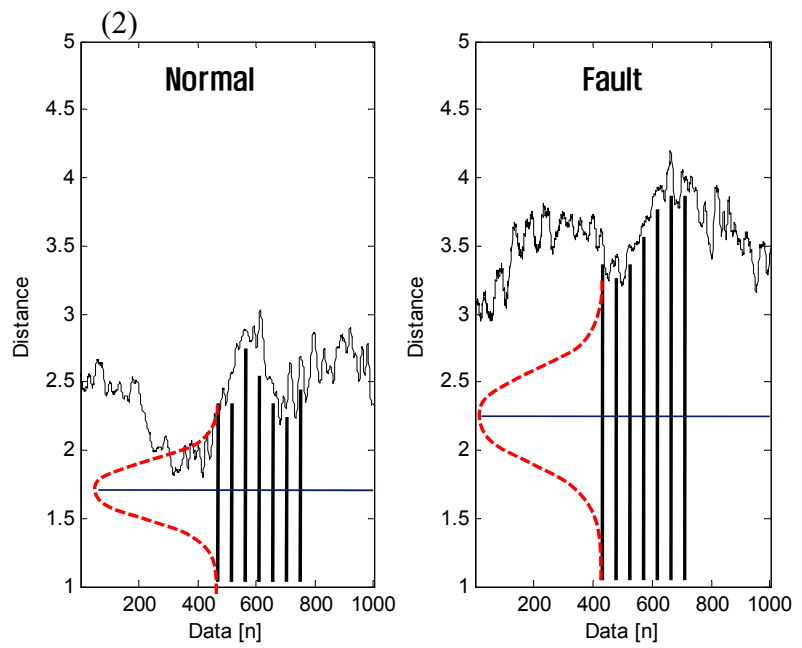

Figure 1. Fuzzy membership function for fault diagnosis.

\section{Experimental Results}

\subsection{Experiment Data for Fault Detection and Isolation}

The current signals for fault detection and isolation were measured based on $600 \mathrm{rpm}$ of the speed and $2 \mathrm{ohms}$ of the fault resistor. The fault resistor is used to mimic the fault of the BLDC motor. The load conditions for fault detection consist of

\begin{tabular}{|c|c|c|c|}
\hline $\mathrm{rpm}$ & Fault resistor & Sampling & Load \\
\hline \multirow{3}{*}{600} & \multirow{3}{*}{$2 \Omega$} & \multirow{3}{*}{$\begin{array}{c}-100[\mathrm{kHz}] \\
-1[\mathrm{~s}]\end{array}$} & No \\
\hline & & & Positive \\
\hline & & & Negative \\
\hline
\end{tabular}
no-, over-, and under-load in data measurement. Table 1 shows the operating conditions of the experimental motor to obtain the fault data. In future research, several conditions of the test motor will be applied to gain the test data such as different speed and fault resistor.

Table 1. Operating conditions in the experiments. 


\subsection{Data Filtering for Data Preprocessing}

\subsubsection{No load $600 \mathrm{rpm}$ fault signals without filtering}

The three phase current of the original data have a fault in each phase as shown in Fig. 2 (a), so it is difficult to find any feature from sum of current signals. Therefore, it is necessary to analyze based on the three-phase current signals. The boxes in Fig. 2 indicate the fault period of each phase of the $600 \mathrm{rpm}$ current signal. However, as shown in the feature extraction results, results are not significantly shown because of noise as shown in Fig. 2 (b). To improve the detecting performance, filtering is necessary in this study.

\subsubsection{No load $600 \mathrm{rpm}$ fault signals with filtering}

In this study, low-pass filtering was employed in the preprocessing stage to avoid the performance degradation caused by the signal noise. There are several methods for filtering, but the simple low-pass filtering was used to remove the high frequency parts as shown in Fig. 3 (a). As inspecting the raw signals after filtering, the specific features appear at the fault points. As shown in Fig. 3 (b), the faults are detected by the Park's vector method. Fault signals generated by $2 \mathrm{ohm}$ have the significant feature for fault detection.

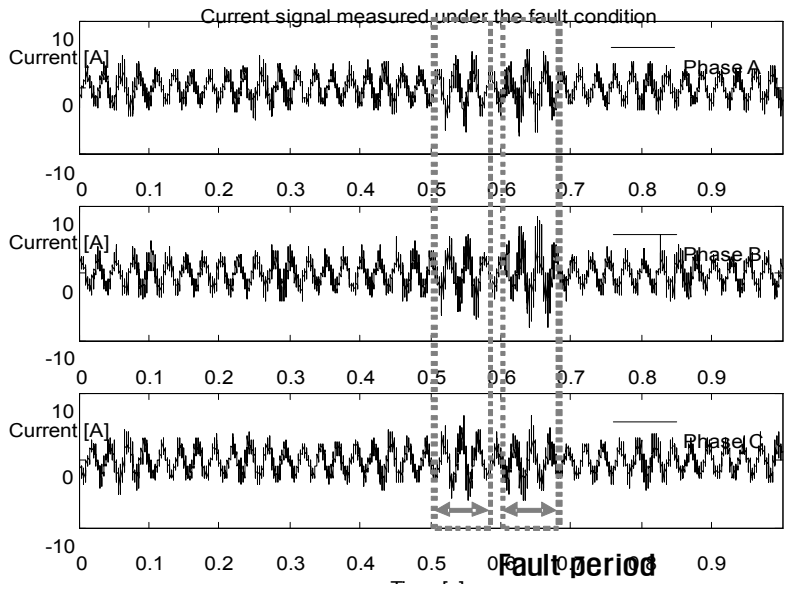

(a) Raw signals of three-phase current without filtering

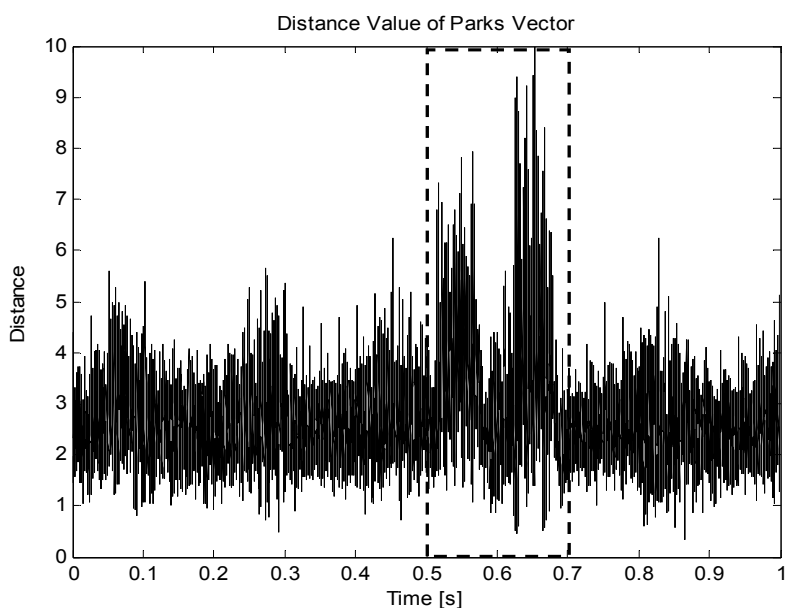

(b) Feature extraction result without filtering

Figure 2. Fault detection without filtering.
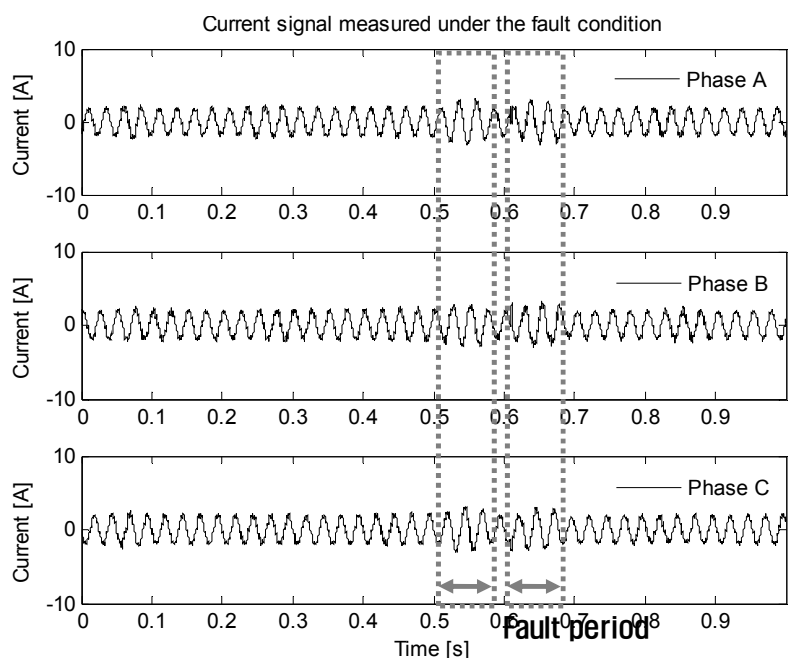

(a) Raw signals of three-phase current with filtering

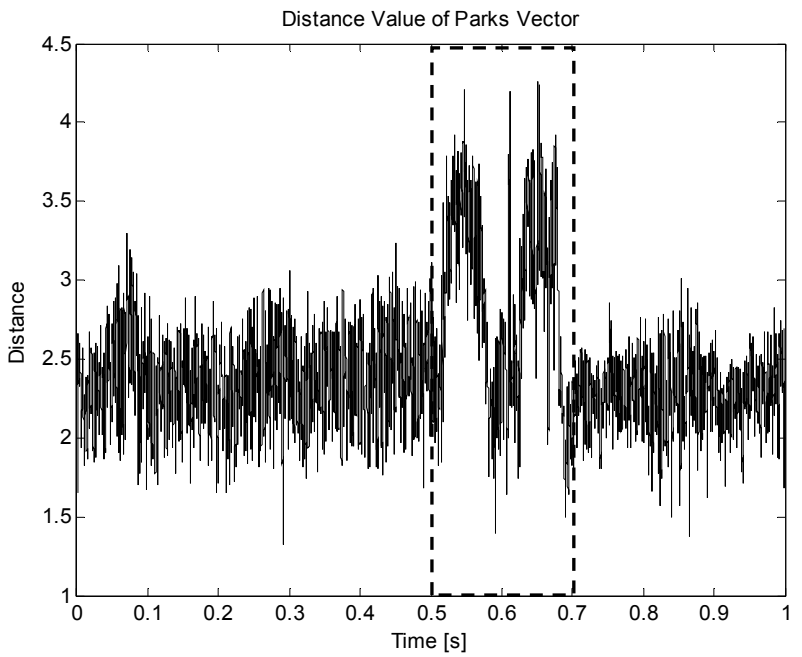

(b) Feature extraction result with filtering

Figure 3. Fault detection with filtering.

\subsection{Results of Fault Detection and Isolation}

\subsubsection{Result of fault detection}

In this study, the signals were measured under the several operating conditions such as $600 \mathrm{rpm}$ of the motor speed, 3 levels of the load, and $2 \mathrm{ohms}$ of the fault resistor. $2 \mathrm{ohm}$ faults can be just useful for feature extraction in this study and 600 rpm data can be used for feature extraction. In this section, 600 rpm singles were employed to generate the results of feature extraction.

To extract features, the Park's vector method mentioned above was applied and the results are shown in Fig. 4 to Fig. 6. As shown in the results, the vector-based approach is better for the several load conditions.

In the future work, 1200, 1800, 2400, and $3000 \mathrm{rpm}$ of the motor speed and 10, 25, 50, and $75 \mathrm{ohm}$ of the fault resistor will be performed for performance validation.

(1) No load

Even though, in the no-load signal, variation of the current signals at the fault points is not significant, there is a distinct 
feature in each phase of the current signal. Vector-based feature extraction from the current signals was used and the result is shown in Fig. 4. As shown in the results, a sharp increase in the signal of the fault points from 0.5 second to 0.7 second is apparently detected. The approach applied in this study can clearly detect the fault from the current signal. The vectorbased approach can give the better performance in feature extraction from no-load data. The best feature can make the best accuracy in the fault isolation.

(2) Positive load

Under the positive loading condition, the amplitude of the current signal is generally increased, so the variation of the current signal at the fault points is very big. This characteristic deduces better performance in feature extraction. However, the fault severity in the high loading condition can be quickly enlarged than that in the no-load condition. Therefore, it is necessary to detect the fault rapidly for stable operation. The approach as shown in Fig. 5 can be properly employed for the purpose. In the results of this case, the vector-based approach shows the good performance.

\section{(3) Negative load}

The amplitude of the current signal under the negative loading condition is decreased. However, the signal at the fault points is shapely varied, so it is well-distinguished. The high frequency parts are pointed up, because the motor is operated under the negative loading condition. As shown in Fig. 6, even though, the variation of the result is a little bit sharp graph, the feature for the fault can be extracted by the method.

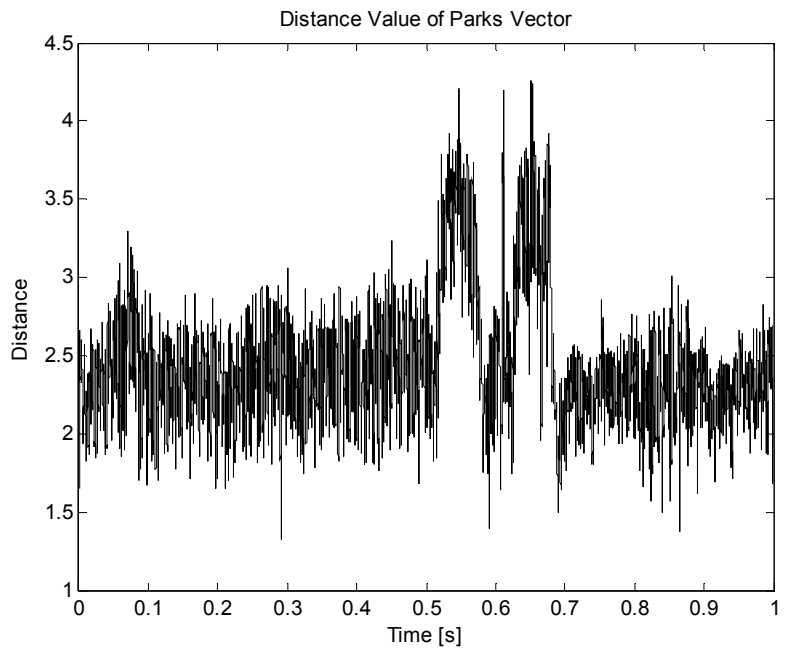

Figure 4. Feature extraction of a no load signal.

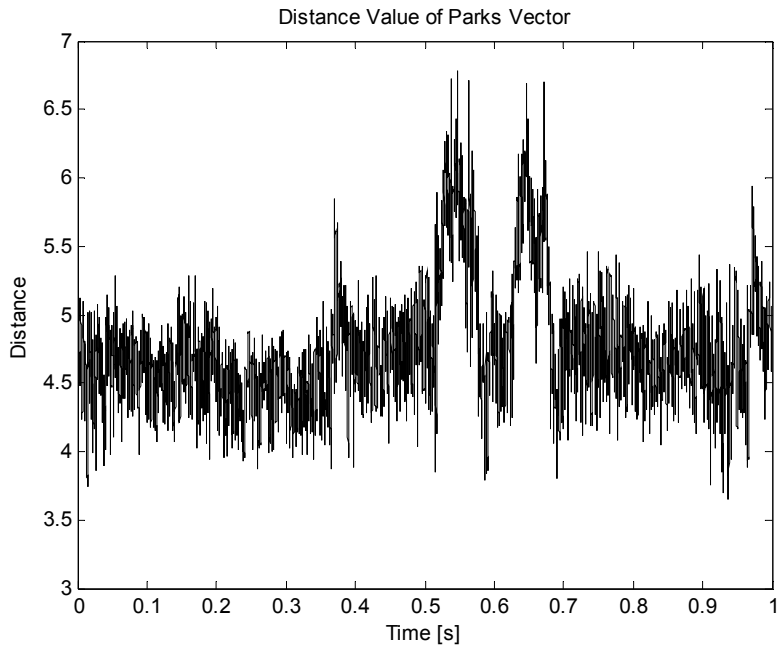

Figure 5. Feature extraction of a positive load signal.

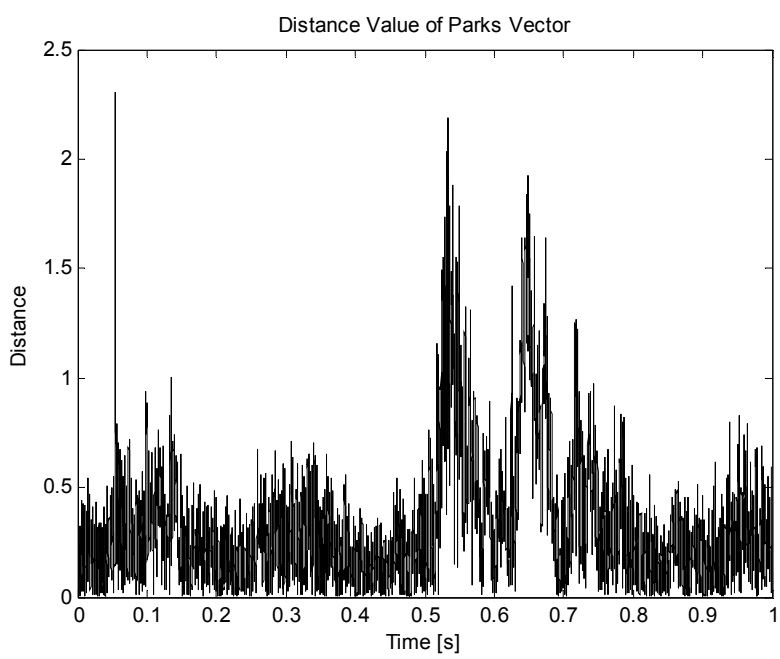

Figure 6. Feature extraction of a negative load signals.

\subsubsection{Result of fault isolation}

To isolate the fault from features extracted by Park's vector, fuzzy similarity was used in this study. Figure 7 shows continuous fuzzy membership functions applied to the 600rpm no load signal for fault isolation. In this processing, one baseline membership function was defined and the rest signal values were compared with the baseline function based on the membership type for fault diagnosis. The final result of the fuzzy similarity is shown in Fig. 8. As shown in the upper graph of Fig. 8, the degree of fuzzy similarity is sharply decreased at the fault points. The fault possibility is expressed as a percentage of the dissimilarity as shown in the bottom graph of Fig. 8. The fault possibility value can be used for fault isolation based on the percentage value whether it is possible or not. In this graph, the threshold is $85 \%$, that is, if the possibility is larger than the threshold, the fault can strongly appear in the BLDC motor. 


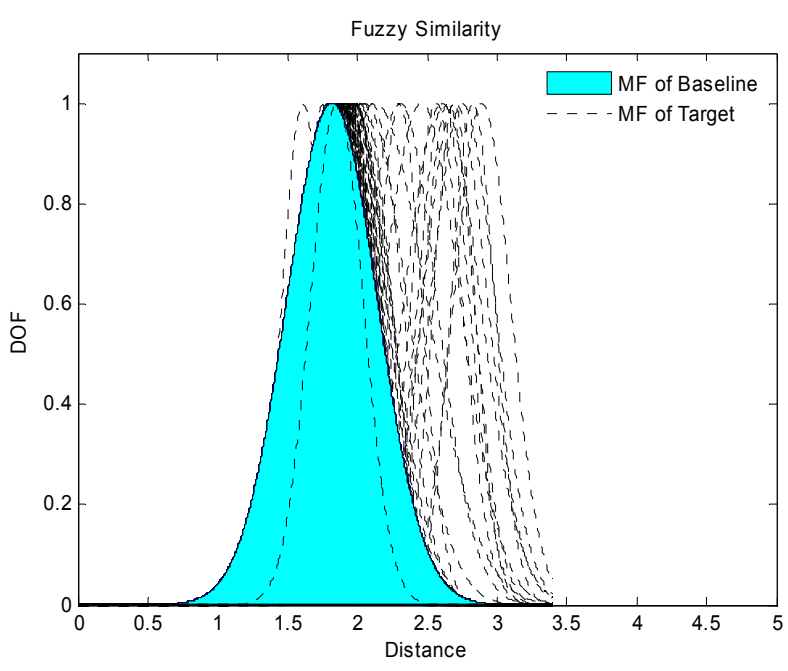

Figure 7. Fuzzy similarity between baseline and targets.
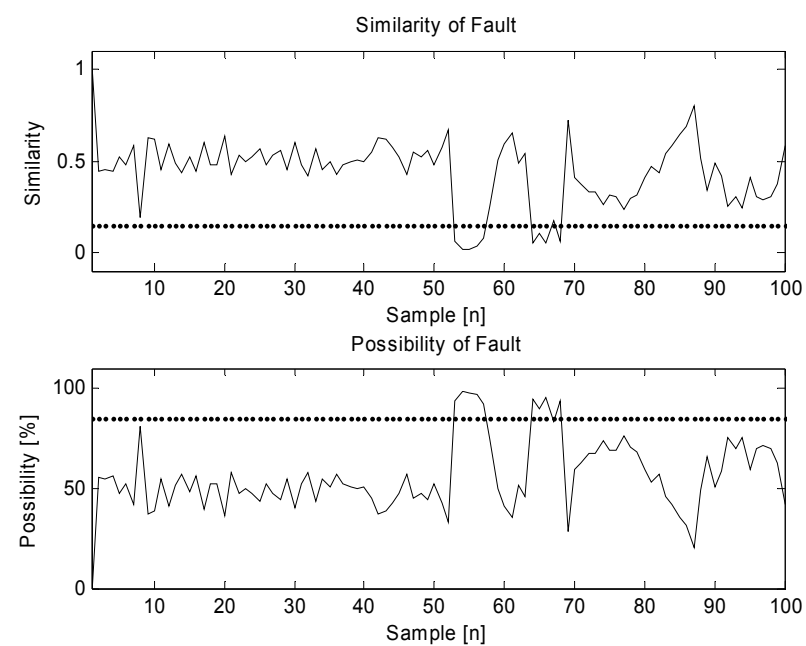

Figure 8 . Result of similarity and fault diagnosis.

\section{Conclusions}

This research described the time-series data mining that extracts features from time-series data such as supplied current signals of BLDC motors. In this study, turn-to-turn short was a target fault that is a severe fault. To extract features from the current signals, Park's vector was used. The approach is useful to obtain the fault feature with the vector-based approach. After extracting the features, fuzzy similarity was employed to isolate the fault based on the features. This method is a simple method without any model for diagnosis.

By using the proposed approach, the faults can be detected and isolated well. However, in this study, specific test conditions were performed but in the future, some more operating conditions will be achieved for performance validation.

\section{References}

[1] P. Vas, Parameter Estimation, Condition Monitoring, and Diagnosis of Electrical Machines, Clarendron Press, Oxford, 1993.

[2] G. B. Kliman and J. Stein, "Induction Motor Fault Detection via Passive Current Monitoring," International Conference in Electrical Machines, Cambridge, MA, pp. 13-17, August 1990.

[3] Y. E. Zhongming and W. U. Bin, "A Review on Induction Motor Online Fault Diagnosis," The Third International Power Electronics and Motion Control Conference (PIEMC 2000), vol. 3, pp. 1353-1358, Aug. 15-18, 2000.

[4] A. J. Marques Cardoso and A. M. S. Mendes, "Semi-converter Fault Diagnosis in DC Motor Drives by Park's Vector Approach," 6th International Conference on Power Electronics and Variable Speed Drives (CP429), pp. 93-98, Nottingham, UK, 23-25 Sep. 1996.

[5] Q. Guo, X. Li, H. Yu, W. Hu, and J. Hu, "Broken Rotor Bars Fault Detection in Induction Motors Using Park's Vector Modulus and FWNN Approach," Lecture Notes In Computer Science, vol. 5264, pp. 809-821, 2008.

[6] A. J. Marques Cardoso, S. M. A. Cruz, J. F. S. Carvalho, and E. S. Saraiva, "Rotor Cage Fault Diagnosis in Three-phase Induction Motors, by Park's Vector Approach," Conference Record of the 1995 IEEE Thirtieth IAS Annual Meeting (IAS '95), vol. 1, pp. 642-646, 8-12 Oct. 1995.

[7] P. Korondi and H. Hashimoto, "Park Vector Based Sliding Mode Control of UPS with Unbalanced and Nonlinear Load," PERIODICA POLYTECHNICA SER. EL. ENG. vol. 43, no. 1, pp. 65-79, 1999.

[8] T. Gadi, R. B. M. Daoudi, and S. Matusiak, "Fuzzy Similarity Measure for Shape Retrieval," Vision Interface '99, TroisRivières, Canada, 19-21 May, 1999.

[9] M. George, "A Fuzzy Similarity Measure Based on the Centrality Scores of Fuzzy Terms," International Conference of the North American Fuzzy Information Processing Society (NAFIPS 2004), pp. 740-744, Alberta, Canada, June 27-30, 2004.

[10] De-Gang Wang, Yan-Ping Meng, and Hong-Xing Li, "A Fuzzy Similarity Inference Method for Fuzzy Reasoning," Computers \& Mathematics with Applications archive, vol. 56, no. 10, pp. 2445-2454, Nov. 2008.

[11]M. Karddouchi and N. Belacel, "Objects Recognition Using SIFT and Fuzzy Similarity Measure," The 20th Annual IS\&T/SPIE Symposium on Electronic Imaging, San Jose, California, USA, Jan. 27-31, 2008. 


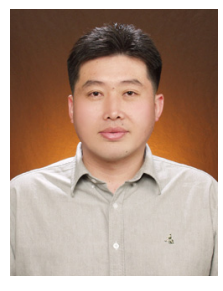

\section{Hyeon Bae}

He received the M.S. and Ph.D. degree in electrical engineering from Pusan National University in 2001 and 2005, respectively. He joined the Intelligent Control Systems Lab as a Post-doctoral fellow at Georgia Institute of Technology from 2007 to 2008. $\mathrm{He}$ is currently a Senior Researcher in the Regional Innovation Agency, Busan Techno-Part. His research interests include intelligent system and control, data mining, systems biology, and bioinformatics.

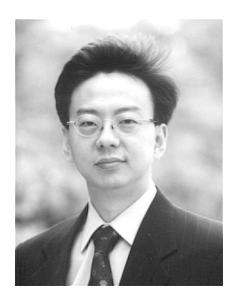

\section{Sungshin Kim}

He received the B.S. and M.S. degrees from Yonsei University, and the Ph.D. degree in electrical engineering from Georgia Institute of Technology, Atlanta, in 1984, 1986, and 1996, respectively. $\mathrm{He}$ is currently an Associate Professor in the School of Electrical and Computer Engineering, Pusan National University. His research interests include intelligent control, fuzzy logic control, manufacturing systems, and data mining.

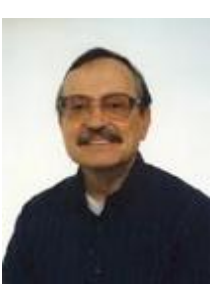

\section{George J. Vachtsevanos}

He attended the City College of New York and received his B.E.E. degree in 1962. He received an M.E.E. degree from New York University and his Ph.D. degree in Electrical Engineering from the City University of New York in 1970. His research focused on adaptive control systems. Since joining the faculty at Georgia Tech, he has been teaching courses and conducting research on intelligent systems, robotics and automation of industrial processes and diagnostics/prognostics of large-scale complex systems. 